\title{
Quantitative assessment of Yersinia enterocolitica in raw pork meat using real time PCR (qPCR) technique
}

\author{
M.A. Stachelska ${ }^{1}$ \\ Lomza State University of Applied Sciences, Akademicka 14, 18-400 Łomża
}

KEY WORDS: real-time PCR, Yersinia enterocolitica, probes, meat, pork

Received: 7 February 2017

Revised: $\quad 17$ May 2017

Accepted: 8 June 2017

${ }^{1}$ Corresponding author:

e-mail: mstachelska@pwsip.edu.pl

\begin{abstract}
The aim of the present study was to develop a highly sensitive and specific TaqMan probe with a set of primers for detection of pathogenic Yersinia enterocolitica strains and to eliminate the pre-PCR enrichment step in the realtime PCR (qPCR). A newly developed qPCR assay which is sensitive and specific for quick and reliable quantitative assessment of $Y$. enterocolitica present in artificially contaminated raw pork meat samples is described. This protocol involves the qPCR method with a TaqMan probe. The primers and probe were designed on the base of locus_tag CH49_3099 gene. This protocol appeared to be reliable for both intended applications: 1. identification and quantification of $Y$. enterocolitica in artificially and naturally contaminated raw pork meat and 2. establishment of growth potentials of different serotypes of $Y$. enterocolitica in raw meat at the usually used storage temperatures. This developed method makes it possible to eliminate the pre-PCR enrichment step enabling for the rapid assessment of meat-related consumer exposure to this pathogen.
\end{abstract}

\section{Introduction}

Yersinia enterocolitica is known to constitute a significant pathogen which possesses a huge impact on the pork production and processing industry in Poland. The Yersinia genus involves commensal microflora of pig intestines and may be easily transfer into carcasses during the improper and non hygienic slaughtering. $Y$. enterocolitica is considered to be responsible for provoking human diseases which includes self-limiting enteritis, acute mesenteric lymphadenitis, septicaemia, Reiter's syndrome and erythema nodosum. These diseases mainly appear as the result of food-borne transmission. Pigs are believed to be the predominant reservoir of $Y$. enterocolitica strains which are pathogenic to humans. Furthermore, this pathogen can grow at low temperatures (even $0{ }^{\circ} \mathrm{C}$ ) and survive at a wide $\mathrm{pH}$ range (5.0-9.6). Consumption of contaminated meat products constitutes the main route for $Y$. enterocolitica infections (Bolton et al., 2013). In Europe this pathogen is defined as the most common one provoking food-borne yersiniosis (Martinez, 2010). There is more non-pathogenic than pathogenic Yersinia strains identified in raw pork meat. Also, there is still a lack of reliable and rapid methods to identify $Y$. enterocolitica since the conventional plating methods are not typically selective for Yersinia because they encourage the growth of other members of the family Enterobacteriaceae. Moreover, they are usually very laborious and time-consuming (Vázlerová and Steinhauserová, 2006; Van Damme et al., 2010). Real-time polymerase chain reaction (PCR) possesses the potential to cope with the significant limitations of conventional plating methods enabling the elimination of the pre-PCR enrichment step. 
The main aim of this study was to develop a highly sensitive and specific TaqMan probe with a set of primers and to eliminate the pre-PCR enrichment step by increasing the reliability of CH49_3099 gene-based real-time PCR (qPCR) used for identification and quantification of enteropathogenic $Y$. enterocolitica strains in raw pork meat. Furthermore, the growth potential in raw pork meat of pathogenic serotypes was assessed at two common storage temperatures.

\section{Material and methods}

\section{Bacterial strains and growth conditions}

In this study, 16 pathogenic Yersinia strains were examined (Table 1). They are considered to be the most frequent serotypes connected with human and animal diseases. Apart from Y. enterocolitica strains there were also examined Yersinia pseudotuberculosis strains.

These strains were incubated overnight at $30{ }^{\circ} \mathrm{C}$ on Brain-Heart Infusion broth (BHI) (Oxoid, Basingstoke, UK) and on Trypton Soya Agar (TSA) (Oxoid, Basingstoke, UK). The total number of viable cells was assessed by preparing 10 -fold serial dilutions of bacterial suspension, plating them on BHI agar (Oxoid, Basingstoke, UK), cultivating overnight at $30{ }^{\circ} \mathrm{C}$ and counting the colony forming units (CFU).

Table 1. A record of Yersinia enterocolitica strains involved in inclusivity assay

\begin{tabular}{|c|c|c|}
\hline Species of Yersinia & Original strain no. & Collection \\
\hline Y. enterocolitica & YE1 & Ghent University, Belgium \\
\hline Y. enterocolitica & YE3 & Ghent University, Belgium \\
\hline Y. enterocolitica & YE5 & Ghent University, Belgium \\
\hline Y. enterocolitica & YE7 & Ghent University, Belgium \\
\hline Y. enterocolitica & YE165 & Ghent University, Belgium \\
\hline Y. enterocolitica & KNG22703 & Ghent University, Belgium \\
\hline Y. enterocolitica & $2516-87$ & Ghent University, Belgium \\
\hline Y. enterocolitica & 8081 & Ghent University, Belgium \\
\hline Y. enterocolitica & WA & Ghent University, Belgium \\
\hline Y. enterocolitica & W22703 & Ghent University, Belgium \\
\hline $\begin{array}{l}\text { Y. enterocolitica subsp. } \\
\text { palearctica }\end{array}$ & $105.5 R(r)$ & Ghent University, Belgium \\
\hline $\begin{array}{l}\text { Y. enterocolitica subsp. } \\
\text { palearctica }\end{array}$ & Y11 & Ghent University, Belgium \\
\hline $\begin{array}{l}\text { Y. enterocolitica subsp. } \\
\text { palearctica }\end{array}$ & 8081 & Ghent University, Belgium \\
\hline Y. pseudotuberculosis & IP32979 & Ghent University, Belgium \\
\hline Y. pseudotuberculosis & IP32981 & Ghent University, Belgium \\
\hline Y. pseudotuberculosis & IP32918 & Ghent University, Belgium \\
\hline
\end{tabular}

The qPCR assay for detection and quantification of enteropathogenic Yersinia spp. in naturally contaminated pork meat

A total of 50 raw pork samples taken from pigs from two slaughterhouses located in the eastern part of Poland were examined for the presence of the pathogenic species of Yersinia spp. by using locus_tag CH49_3099 gene targeted qPCR. The sampling was carried out according to the European Commission microbiological sampling guidelines (EC, 2006). Pork samples from carcasses were cut aseptically immediately after evisceration, placed into sterile plastic bags and delivered to the laboratory under chilled conditions. Pork samples were aseptically cut into small pieces, and $10 \mathrm{~g}$ of samples were put into sterile stomacher bag. Samples were homogenized with $90 \mathrm{ml}$ of tryptic soya broth (Oxoid, Basingstoke, UK) for $2 \mathrm{~min}$ in a stomacher (Colworth Stomacher 400, Seward Limited, London, UK). Then they were stored at $4{ }^{\circ} \mathrm{C}$ and subjected to DNA isolation within $4 \mathrm{~h}$ after collection.

\section{Extraction of genomic DNA from bacterial cells}

A 10-ml portion of overnight bacterial growth in BHI was centrifuged at $11700 \mathrm{~g}$ for $10 \mathrm{~min}$, washed in $5 \mathrm{ml}$ of $0.1 \times \mathrm{SSC}(1 \times \mathrm{SSC}$ is $0.15 \mathrm{M} \mathrm{NaCl}, 0.015 \mathrm{M}$ sodium citrate), suspended in $1 \mathrm{ml}$ of $0.01 \mathrm{M}$ sodium phosphate buffer in $20 \%$ sucrose ( $\mathrm{pH} 7.0$ ) with lysozyme $\left(2.5 \mathrm{mg} \cdot \mathrm{ml}^{-1}\right)$ and incubated for $45 \mathrm{~min}$ at $37^{\circ} \mathrm{C}$. A 9-ml portion of lysis buffer $(10 \mathrm{mM}$ Tris-hydrochloride ( $\mathrm{pH} 8.0$ ), $1 \mathrm{mM}$ EDTA, $500 \mu \mathrm{g}$ of pronase $\mathrm{B}$ per $\mathrm{ml}, 1 \%$ sodium dodecyl sulphate) was then added. After additional $30 \mathrm{~min}$ at $37^{\circ} \mathrm{C}$, the samples were deproteinized by extraction with phenol and chloroform, and the nucleic acids were precipitated with ethanol. The samples were suspended in $10 \mathrm{mM}$ Tris-hydrochloride ( $\mathrm{pH}$ 8.0), $1 \mathrm{mM}$ EDTA and stored at $4{ }^{\circ} \mathrm{C}$. The concentration of DNA $\left(\mathrm{ng} \cdot \mu \mathrm{l}^{-1}\right)$ was measured by spectrophotometer (Evolution 220, Thermo Fisher Scientific, Waltham, MA, USA). The mass $(\mathrm{M})$ of 1 genomic molecule was counted on the base of the equation $\mathrm{M}=\mathrm{n} \times\left(1.01 \times 10^{-21} \mathrm{~g} \cdot \mathrm{bp}^{-1}\right)$; for Yersinia $4.616 \times 10^{6} \mathrm{bp}$. The quantified DNA (number of genomic copies per $\mu$ l) was serially diluted in water and underwent qPCR according to the conditions specified below.

\section{Inoculating meat samples}

Raw pork meat negative for enteropathogenic Yersinia was divided into subsamples containing $1 \mathrm{~g}$ of raw pork meat and $9 \mathrm{ml}$ of BHI, and inoculated with the tested bacterial strain to a final concentration 
of approximately $10^{5}, 10^{4}, 10^{3}$ and $10^{2} \mathrm{CFU} \cdot \mathrm{ml}^{-1}$. DNA was isolated directly from these samples of meat using Syngen Food DNA Mini Kit (Syngen Biotech, Wrocław, Poland). The growth of $Y$. enterocolitica strains in the contaminated meat samples was studied at two different temperatures: $4{ }^{\circ} \mathrm{C}$ and $10{ }^{\circ} \mathrm{C}$ for $72 \mathrm{~h}$, then they were subjected to DNA isolation. Two different temperatures were applied to assess the growth rate of $Y$. enterocolitica strains. The qPCR was carried out in the following steps.

\section{DNA isolation from control meat samples and meat samples inoculated with Yersinia spp. cells}

DNA was isolated from $10 \mathrm{ml}$ of bacterial suspension containing $1 \mathrm{~g}$ of raw pork meat and $9 \mathrm{ml}$ of BHI using Syngen Food DNA Mini Kit (Syngen Biotech, Wrocław, Poland). It enabled to achieve $350 \mu \mathrm{l}$ of DNA extract. DNA concentration was measured by spectrophotometer (Evolution 220; Thermo Fisher Scientific, Waltham, MA, USA).

\section{The qPCR assay}

The primer/probe set targeting locus_tag $\mathrm{CH} 49$ 3099 gene was designed on the base of 50-nucleotide sequence GACGATACCTTGTATAGCAATCTATTTAGCACTGATGTGTCGGTTCCGG specific for $Y$. enterocolitica species. The sequence of the gene was provided by GenBank (www.ncbi.nlm. nih.gov/Genbank/; Accession No. CP009846.1). Sequences unique for $Y$. enterocolitica were compared with those of closely related strains. The primer/ probe set was designed using Primer Express Software v3.0 (Applied Biosystems, Foster City, CA, USA). The set was validated using NCBI BLAST (Basic Local Alignment Search Tool: www.ncbi.nlm. nih.gov/blast/). The sequences were as follows: forward primer 5'-GACGATACCTTGGTATAGC-3'; reverse primer 5'-ATAGCTGATGACTTTAT-3'; probe 5'-FAM-CCGGAACCGACACATCAGTGCTAAATAGAT-3'-MGB-NFQ. The amplicon size was 66 bases long. The oligonucleotides were synthesised and purchased from Eurofins Genomics (Ebersberg, Germany).

The PCR mixture contained $1 \times$ TaqMan Universal Master Mix (Syngen, Cambridge, UK), $300 \mathrm{nM}$, $600 \mathrm{nM}$ and $900 \mathrm{nM}$ of reverse and forward primers respectively, and $200 \mathrm{nM}$ of FAM-MGB-NFQ labelled probe and $5 \mu 1$ of template DNA in total volume of $20 \mu \mathrm{l}$. A non-template control (NTC) contained $5 \mu 1$ of water instead of DNA and was included in each run. The qPCR cycling parameters were the following: 1 cycle of initial denaturation of DNA at $95{ }^{\circ} \mathrm{C}$ for 10 min followed by 40 cycles of amplification with denaturation at $95^{\circ} \mathrm{C}$ for $15 \mathrm{~s}$, and annealing and extension at $60{ }^{\circ} \mathrm{C}$ for $1 \mathrm{~min}$. All qPCR assays were performed in the Stratagene Mx3005P thermocycler (Agilent Technologies, Santa Clara, CA, USA).

\section{Statistical analysis}

Results of quantitative and qualitative testing were recorded in an Excel programme. The analysis of descriptive data was carried out using Stata 8.0 software (Stata Corporation, College Station, TX, USA). Comparison of enumeration results was performed using Poisson regression.

\section{Results}

\section{Inclusivity and exclusivity of newly designed qPCR}

With the exception of $Y$. enterocolitica there were not any positive reactions for the probe/primers set with unrelated microorganisms. The assay proved to be in $100 \%$ sensitive and specific towards the pathogenic species of $Y$. enterocolitica. For inclusivity and exclusivity tests of the designed sequences, qPCR was carried out on 22 strains (Tables 1 and 2).

\section{Optimization of qPCR conditions in pork meat and assessing the linearity and quantification range}

The optimalization of qPCR method conditions was conducted on the basis of the study of Najdenski et al. (2012). The concentrations of forward and reverse primers in the amount of $300 \mathrm{nM}$, $600 \mathrm{nM}$ and $900 \mathrm{nM}$ together with $200 \mathrm{nM}$ of FAMMGB-NFQ labelled probe were analysed using genomic DNA isolated from $Y$. enterocolitica strain 8081 serotype O:8 (Accession No. in GeneBank: CP009846.1). The DNA concentration was $10-10^{5}$ of genomic copies per reaction. This strain was used as template. The TaqMan Universal Master Mix (Syngen, Cambridge, UK) was used as PCR mix. The optimal concentration of forward and reverse primers was $900 \mathrm{nM}$ and was applied in the subsequent studies. The standard curve made on the base of these reaction conditions indicated a linear relationship $\left(\mathrm{R}^{2}=0.999\right)$ between log input DNA and the amplification cycle (regression equation $y=-3.73 x+42.92)$. Such results indicates that the qPCR has a range of linear quantification from 10 to $10^{5}$ genomic copies per reaction. 
Table 2. Partial locus_tag CH49_3099 gene sequence alignment of 6 strains of Yersinia with database sequence indicating sequence differences

\begin{tabular}{|c|c|c|c|}
\hline Strain (GeneBank Accession No.) & Forward primer 5'-3' & Probe 3'-5' & Reverse primer 3'-5' \\
\hline Y. enterocolitica (СР009846.1) & GACGATACCTTGGTATAGC & ATCTATTTAGC & STCATCAGCTAT \\
\hline Y. kristensenii (CP & & & \\
\hline Y. inte & & & \\
\hline (AE009952.1) & CACC & $\mathrm{CCC}$ & SAGGGCGA \\
\hline Y. pseudotuberculosis (CP009786.1) & GGTGCTGGTTTCATGGCTG & CCCAATAACAGCAGC & TTGTACATG \\
\hline Y. rohdei (CP009787.1) & TAAAGACAACCAACTGGCA & CT TT CCAACATTCCGGT TT CTGGC AACCTT & GATACTCTCCGCGACAA \\
\hline
\end{tabular}

\section{DNA isolation from pork meat samples}

DNA was isolated from 10 pork meat samples which were Yersinia-negative. The isolation was performed according to Syngen Food DNA Mini Kit (Syngen Biotech, Wrocław, Poland). The achieved DNA samples were inoculated with $10^{5}$ genomic copies from $Y$. enterocolitica 8081 DNA. The qPCR with the TaqMan Universal Master Mix (Syngen, Cambridge, UK) showed the quantification cycle values (Cq which is the cycle number where the fluorescence passes the threshold line) being in the range from 22 to 27 , whereas the $\mathrm{Cq}$ value of $10^{5}$ genomic copies from $Y$. enterocolitica 8081 amounted 22.2. Such results show that the majority of pork meat samples indicated the inhibition of the PCR reaction.

\section{Assessing the efficiency of the TaqMan Environmental Master Mix}

The designed primer/probe set targeting locus_tag CH49_3099 gene specific for Y. enterocolitica was examined in the qPCR with application of the TaqMan Environmental Master Mix (Syngen, Cambridge, UK). The standard curve created on the base of genomic DNA dilutions indicated a linear relationship $\left(\mathrm{R}^{2}=0.999\right)$ between log input DNA and the quantification cycle (regression equation $\mathrm{y}=-3.91 \mathrm{x}+43.08)$. The $\mathrm{Cq}$ values from the DNA coming from 10 pork meat samples inoculated with $10^{3}$ genomic copies of $Y$. enterocolitica 8081 changed not very significantly (30.75-31.43) and were comparable to $10^{3}$ copies of the pure genomic DNA coming from the bacterial culture (31.39). Such results proved that the TaqMan Environmental Master Mix was less sensible to the inhibition caused by meat components in comparison with the TaqMan Universal Master Mix. Due to its high efficiency, the TaqMan Environmental Master Mix was applied in all further studies.

\section{Exclusivity of the assay}

The aim of the research was also to confirm the exclusivity of locus_tag CH49_3099 gene base in the qPCR. DNA coming from pure bacterial cultures of 5 Yersinia species involving Y. kristensenii, Y. inter- media, Y. pestis, Y. pseudotuberculosis and Y. rohdei were examined (Table 2). None of qPCR reactions carried out on the above species gave a positive result. This proves the specificity of the designed primer/probe set only for $Y$. enterocolitica species.

\section{Inclusivity of the assay}

The inclusivity of the designed primer/probe set with the TaqMan Environmental Master Mix was examined by using bacterial genomic DNA $\left(1 \times 10^{4}\right.$ molecules) coming from Y. enterocolitica strains including original strain numbers YE1, YE3, YE5, YE7, YE165, KNG22703, 2516-87, 8081, WA, W22703, 105.5 and Y11 as template spiked into $5 \mu \mathrm{l}$ of meat DNA. All samples showed positive reactions with $\mathrm{Cq}$ values in the range from 27 to 28 which stays in the high correlation with the Cq value (27.68) achieved from $10^{4}$ genomic copies of the standard $Y$. enterocolitica 8081 . The negative control gave no positive reaction.

\section{Quantitative assessment of $Y$. enterocolitica in pork meat}

The designed primer/probe set for the qPCR was used for raw pork meat samples inoculated with bacterial cells of various serotypes of $Y$. enterocolitica (Table 1). As the infective dose of $Y$. enterocolitica for humans amounts $10^{4} \mathrm{CFU}$, pork meat samples were inoculated with various concentrations of this strain which involved: $1 \times 10^{5}, 1 \times 10^{4}$, $1 \times 10^{3}, 1 \times 10^{2}, 1 \times 10^{1} \mathrm{CFU} \cdot \mathrm{g}^{-1}$. The differences between theoretically expected log genome copies and detected log genome copies in $1 \mathrm{~g}$ of meat are shown in Table 3.

The amount of theoretically expected DNA copies is based on the real concentration of inoculated bacteria examined after plating of bacterial solution which was applied for inoculating of the pork meat samples. In case when relatively high concentrations of bacteria were inoculated (in the range from $10^{3}$ to $10^{5} \mathrm{CFU} \cdot \mathrm{g}^{-1}$ ), identified DNA copy numbers stayed well with the correlation with the expected number based on plate count. The difference changed from 0.93 to $-0.74 \log$ units. The inoculation with 
Table 3. Differences between theoretically expected log DNA copies and detected log DNA copies in $1 \mathrm{~g}$ of meat after inoculating of meat samples with different concentration of different species of Yersinia enterocolitica

\begin{tabular}{|c|c|c|c|c|}
\hline \multirow{2}{*}{$\begin{array}{l}\text { Strain } \\
\text { of Yersinia enterocolitica }\end{array}$} & \multicolumn{4}{|c|}{$\Delta \log C F U \cdot g^{-1}$} \\
\hline & $\sim 5 \log / g^{b}$ & $\sim 4 \log / g^{b}$ & $\sim 3 \log / g^{b}$ & $\sim 2 \log / g^{b}$ \\
\hline Y. enterocolitica YE1 & $0.12^{\mathrm{a}}$ & 0.09 & 0.07 & -0.53 \\
\hline Y. enterocolitica YE3 & 0.93 & 0.62 & -0.28 & -0.58 \\
\hline Y. enterocolitica YE5 & 0.33 & 0.04 & 0.18 & -0.08 \\
\hline Y. enterocolitica YE7 & 0 & 0.03 & -0.48 & 0.10 \\
\hline Y. enterocolitica YE165 & 0.62 & 0.59 & 0.16 & 0.12 \\
\hline Y. enterocolitica KNG22703 & 0.55 & 0.48 & 0.32 & 0.15 \\
\hline Y. enterocolitica 2516-87 & 0.67 & 0.43 & 0.38 & 0.18 \\
\hline Y. enterocolitica 8081 & -0.74 & -0.47 & -0.58 & -0.55 \\
\hline Y. enterocolitica WA & 0.62 & 0.36 & 0.28 & -0.28 \\
\hline Y. enterocolitica W22703 & 0.58 & 0.43 & 0.38 & -0.46 \\
\hline $\begin{array}{l}\text { Y. enterocolitica subsp. } \\
\text { palearctica } 105.5 R\end{array}$ & 0.53 & 0.34 & 0.23 & -0.28 \\
\hline $\begin{array}{l}\text { Y. enterocolitica subsp. } \\
\text { palearctica Y11 }\end{array}$ & 0.6 & 0.18 & 0.58 & -0.33 \\
\hline $\begin{array}{l}\text { Y. enterocolitica subsp. } \\
\text { palearctica } 8081\end{array}$ & 0.48 & 0.16 & 0.51 & -0.37 \\
\hline
\end{tabular}

a differences (expressed in $\triangle \log C F U \cdot \mathrm{g}^{-1}$ of meat) between theoretically expected log DNA copies and detected log DNA copies in $1 \mathrm{~g}$ of meat after inoculating of meat samples with different concentration of different species of Yersinia enterocolitica were based on the real concentration of inoculated bacteria examined after plating of bacterial solution which was applied for inoculating of pork meat samples; ${ }^{\mathrm{b}}$ spiking level based on turbidity

relatively low amount $10^{2} \mathrm{CFU} \cdot \mathrm{g}^{-1}$ gave a positive qPCR reaction with detected difference of copy numbers in the range from $\log 0.12$ to $\log -0.58$. The Cq changed from 37.9 to 36.3 , which stays in correlation with a concentration ranged from 6.9 to 68 genomic copies/g of meat. There was not any amplification found in any of the negative control samples. Such results indicate that the qPCR system possesses the linear quantification in the range from $10^{3}$ to $10^{5} \mathrm{CFU} \cdot \mathrm{g}^{-1}$ and a detection limit less than $10^{2} \mathrm{CFU} \cdot \mathrm{g}^{-1}$.

\section{Growth of $Y$. enterocolitica in raw meat during storage at various temperatures assessed by qPCR}

To assess a rate of multiplication of different $Y$. enterocolitica strains which belong to the serotypes O:8, O:9 and O:3, the meat samples were artificially contaminated and stored for $48 \mathrm{~h}$ at $4{ }^{\circ} \mathrm{C}$ (optimal) and $10{ }^{\circ} \mathrm{C}$ (suboptimal) storage regimes in the meat chain. The contamination doses included $1 \times 10^{4}$ and $1 \times 10^{1} \mathrm{CFU} \cdot \mathrm{g}^{-1}$. After incubation the number of genomic copies was assessed using qPCR. The multiplication of bacteria stored at $4{ }^{\circ} \mathrm{C}$ occurred for all three examined serotypes but was the most significant for the most pathogenic sero- type $0: 8$ strain. When meat was inoculated with a high number of cells in amount of $1 \times 10^{4} \mathrm{CFU} \cdot \mathrm{g}^{-1}$ of this strain, an increase of 3 and $4 \log$ was found after incubation at $4{ }^{\circ} \mathrm{C}$ and $10{ }^{\circ} \mathrm{C}$. When meat samples were inoculated with a low number of cells in amount of $1 \times 10^{1} \mathrm{CFU} \cdot \mathrm{g}^{-1}$ of this strain, an increase of $5 \log$ was found after incubation at $10^{\circ} \mathrm{C}$ for $48 \mathrm{~h}$. The weakest growth was assessed for the serotype $\mathrm{O}: 3$ strain at $4{ }^{\circ} \mathrm{C}$ and $10{ }^{\circ} \mathrm{C}$ using an inoculation doses $1 \times 10^{4}$ and $1 \times 10^{1} \mathrm{CFU} \cdot \mathrm{g}^{-1}$. No amplification was found in the negative control samples.

\section{Assessment of natural contamination of raw meat with $Y$. enterocolitica by qPCR}

A number of 35 raw pork samples were examined for the presence of pathogenic $Y$. enterocolitica by application of the qPCR assay described earlier. Three samples were found to be positive. The detected numbers of pathogenic $Y$. enterocolitica cells amounted $4.2 \times 10^{2} ; 3.3 \times 10^{2}$ and $1 \times 10^{2}$ copies per $\mathrm{g}$ of meat. They were positive for locus_tag CH49_3099 gene.

\section{Discussion}

Over the last few years many classical microbiological and immunological methods applied to identify pathogenic Yersinia have been improved using DNA-based methods. Some of them involve in vitro amplification in combination with direct hybridization techniques. There is a number of modifications involving enrichment, filtration, centrifugation and absorption which have been applied to concentrate and separate $Y$. enterocolitica strains in natural samples of meat. However, it is still not possible to directly isolate all the pathogenic serotypes (Wauters et al., 1987; Weynants et al., 1996; Fredriksson-Ahomaa and Korkeala, 2003).

Nowadays PCR is widely used in in vitro amplification method because it is able to selectively identify many microorganisms. Conventional PCR with primers coming from the chromosomal ail gene have been used for successful identification of $Y$. enterocolitica in food matrices including pork meat, beef meat and cheese (Boyapalle et al., 2001; Thisted Lambertz et al., 2008).

The qPCR assays, especially those which involve TaqMan probes, are more exclusive and less timedemanding in comparison with the conventional PCR. There is still a growing number of newly developed qPCR assays to identify enteropathogenic $Y$. enterocolitica in different food matrices applying a TaqMan probe. These methods enable to obtain the results 
for the possible consumer exposures to pathogenic $Y$. enterocolitica and prevent from disease outbreaks (Stenkova et al., 2008; Bonardi et al., 2014).

In our study we developed an optimized, fast, sensitive and specific qPCR assay with the application of a TaqMan probe to identify and qualitatively assess the number of pathogenic $Y$. enterocolitica in contaminated raw meat samples. The protocol possesses practical significance because it involves optimized concentrations of primers, a kit for DNA extraction from meat samples and the TaqMan Environmental Master Mix.

It should be note that there is still a considerable number of recorded cases of food-borne infections provoked by consumption of meat contaminated with pathogenic strains of $Y$. enterocolitica. The ability to quickly identify the pathogenic strains in food matrices is essential for epidemiological assessment. However, there is not a traditional culture method which gives the reliable results. The pathogenic yersiniae constitute a high public health risk as they are able to grow in refrigerated and vacuum-packed meat (Tauxe et al., 1987; Fredriksson-Ahomaa et al., 2007). Typical heat treatment of meat causes the inactivation of $Y$. enterocolitica strains. It means that pathogenic cells of $Y$. enterocolitica can be transferred to people's digestive tract through the consumption of contaminated post-pasteurized meat or contaminated raw meat (Fosse et al., 2008).

In our study we successfully developed a qPCR assay to detect pathogenic $Y$. enterocolitica strains present in contaminated raw meat samples without the need of enrichment procedure for molecular detection. This is a very rapid and sensitive method which enable to detect and quantify pathogenic $Y$. enterocolitica strains present in pork meat. The method is specific and reliable because the examined non-pathogenic $Y$. enterocolitica strains were all negative for locus_tag CH49_3099 gene-targeted qPCR.

The isolation of very little amount of pathogenic $Y$. enterocolitica strains in food products rich in inhibitory contaminants is very hard to carry out and may give false-negative results when the food matrices are examined for the presence of this pathogenic bacteria (Van Damme et al., 2013a). It is known that direct isolation with the application of selective media together with long-lasting enrichment steps requires a lot of time and they are very seldom successful (Thisted Lambertz et al., 2007). In our research, the conventional culturing methods were not successful and did not enable to isolate pathogenic $Y$. enterocolitica strains from meat samples which were positive for locus_tag CH49_3099 gene-targeted qPCR. This is probably caused by the fact that there was not any enrichment step involved or Yersinia cells were in a non-cultivable condition. For this reason in our study we applied artificially contaminated meat samples which were treated as a standard to assess the quantitative rates of our investigations.

There is a high number of studies which assess the appearance of pathogenic $Y$. enterocolitica strains in different food products involving meat and meat products. Such studies usually give only information about the qualitative detection of $Y$. enterocolitica in samples taken at slaughter. There is still very little information on the quantitative results relating to this pathogen and the molecular procedures for the quantitative assessment usually involve the enrichment step to achieve a sufficient number of cells to be detected (Van Damme et al., 2013b). The samples, in which quantitative research is carried out, involve pork and pork products, beef and beef products, raw milk, fish, carrots and water (Gürtler et al., 2005; Thisted Lambertz et al., 2008).

As $Y$. enterocolitica is a potential threat for human health because it may grow in the food products to levels sufficient to cause sickness, their presence in food products should be monitored by a quantitative PCR test. Sickness can result not only from the consumption of the food (which in this case appears unlikely as pork meat is normally cooked sufficiently to kill any Yersinia), but through the handling of the meat.

The inclusivity and exclusivity tests prove the specificity and reliability of locus_tag CH49_3099 gene-targeted qPCR system. This is the gene which is chromosomally encoded and stably inherited in opposition to the plasmid encoded virulence factors which might be lost during microbiological procedures. Our q PCR assay gave negative results for the presence of Y. pseudotuberculosis (Galindo et al., 2011).

\section{Conclusions}

This study presents the newly developed TaqMan real-time PCR (qPCR) method which enables to detect and quantify a number of pathogenic Yersinia enterocolitica strains in contaminated raw meat samples which were stored at various temperatures. The growth of $Y$. enterocolitica strains proved to stay in close dependence with the serotype, storage temperatures and inoculation dose. 
Further investigation is required in order to examine qPCR-positive meat samples with the isolation of pathogenic $Y$. enterocolitica strains. Furthermore, this method will deliver more quantitative results on the presence of $Y$. enterocolitica in meat and meat products. What is more, it will also support with the risk assessment and the establishment of consumer protection policy. However, this test may have some limitations. It should be noted that it will detect DNA of cells which are both viable and dead. Only viable bacteria will be a threat to public health. The other consideration is the cost of such a test for the meat industry. It is regarded to be relatively cost effective for the manufacturer of pork products and such obstacle should also be the subject of thinking.

\section{Acknowledgements}

The research was supported by the founds granted by Polish Ministry of Science and Higher Education within Project No. BDS-15/ITŻiG/10/2013.

\section{References}

Bolton D.J., Ivory C., McDowell D., 2013. A small study of Yersinia enterocolitica in pigs from birth to carcass and characterization of porcine and human strains. Food Control 33, 521-524, https://doi.org/10.1016/j.foodcont.2013.03.039

Bonardi S., Alpigiani I., Pongolini S., Morganti M., Tagliabue S., Bacci C., Brindani F., 2014. Detection, enumeration and characterization of Yersinia enterocolitica 4/0:3 in pig tonsils at slaughter in Northern Italy. Int. J. Food Microbiol. 177, 9-15, https://doi. org/10.1016/j.ijfoodmicro.2014.02.005

Boyapalle S., Wesley I.V., Hurd H.S., Reddy P.G., 2001. Comparison of culture, multiplex, and 5' nuclease polymerase chain reaction assay for the rapid detection of Yersinia enterocolitica in swine and pork products. J. Food Protect. 64, 1352-1361, https://doi.org/10.4315/0362-028X-64.9.1352

EC, 2006. Guidance Document of the Commission of the European Communities on official controls, under Regulation (EC) No. 882/2004, concerning microbiological sampling and testing of foodstuffs

Fosse J., Seegers H., Magras C., 2008. Foodborne zoonoses due to meat: a quantitative approach for a comparative risk assessment applied to pig slaughtering in Europe. Vet. Res. 39, 01 https://doi.org/10.1051/vetres:2007039

Fredriksson-Ahomaa M., Korkeala H., 2003. Low occurrence of pathogenic Yersinia enterocolitica in clinical, food, and environmental samples: a methodological problem. Clin. Microbiol. Rev. 16, 220-229, https://doi.org/10.1128/CMR.16.2.220229.2003

Fredriksson-Ahomaa M., Stolle A., Stephan R., 2007. Prevalence of pathogenic Yersinia enterocolitica in pigs slaughtered at a Swiss abattoir. Int. J. Food Microbiol. 119, 207-212, https:// doi.org/10.1016/j.ijfoodmicro.2007.07.050
Galindo C.L., Rosenzweig J.A., Kirtley M.L., Chopra A.K., 2011. Pathogenesis of $Y$. enterocolitica and Y. pseudotuberculosis in human yersiniosis. J. Pathog. 2011, 182051, https://doi. org/10.4061/2011/182051

Gürtler M., Alter T., Kasimir S., Linnebur M., Fehlhaber K., 2005. Prevalence of Yersinia enterocolitica in fattening pigs. J. Food Protect. 68, 850-854, https://doi.org/10.4315/0362028X-68.4.850

Martinez P.O., 2010. Prevalence of enteropathogenic Yersinia in pigs from different European countries and contamination in pork production chain. MA Dissertation. Department of Food Hygiene and Environmental Health, Faculty of Veterinary Medicine, University of Helsinki (Finland), URN:ISBN:978-952-10-6393-0, http://hdl.handle.net/10138/19033

Najdenski H., Heyndrickx M., Herman L., Werbrouck H., Van Coillie E., 2012. Quantification of Yersinia enterocolitica in raw milk using qPCR. Vet. Microbiol. 160, 428-434, https://doi. org/10.1016/j.vetmic.2012.06.013

Stenkova A.M., Isaeva M.P., Rasskazov V.A., 2008. Development of a multiplex PCR procedure for detection of Yersinia genus with identification of pathogenic species (Y. pestis, Y. pseudotuberculosis, and Y. enterocolitica). Mol. Genet. Microbiol. Virol. 23, 119-125, https://doi.org/10.3103/S0891416808030038

Tauxe R.V., Wauters G., Goossens V., Van Noyen R., Vandepitte J., Martin S.M., De Mol P., Thiers G., 1987. Yersinia enterocolitica infections and pork. Lancet 329(8542), 1129-1132, https:// doi.org/10.1016/S0140-6736(87)91683-7

Thisted Lambertz S., Granath K., Fredriksson-Ahomaa M., Johansson K.-E., Danielsson-Tham M.-L., 2007. Evaluation of a combined culture and PCR method (NMKL-163A) for detection of presumptive pathogenic Yersinia enterocolitica in pork products. J. Food Prot. 70, 335-340, https://doi.org/10.4315/0362028X-70.2.335

Thisted Lambertz S., Nilsson C., Hallanvuo S., Lindblad M., 2008. Real-time PCR method for detection of pathogenic Yersinia enterocolitica in food. Appl. Environ. Microbiol. 74, 6060-6067, https://doi.org/10.1128/AEM.00405-08

Van Damme I., Berkvens D., Baré J., De Zutter L., 2013a. Influence of isolation methods on the occurrence of plasmid-carrying Yersinia enterocolitica serotype 0:3 in slaughter pig tonsils, faeces and carcass surface swabs. Int. J. Food Microbiol. 164, 32-35, https://doi.org/10.1016/j.ijfoodmicro.2013.03.019

Van Damme I., Berkvens D., Botteldoorn N., Dierick K., Wits J., Pochet B., De Zutter L., 2013b. Evaluation of the ISO 10273:2003 method for the isolation of human pathogenic Yersinia enterocolitica from pig carcasses and minced meat. Food Microbiol. 36, 170-175, https://doi.org/10.1016/j.fm.2013.05.007

Van Damme I., Habib I., De Zutter L., 2010. Yersinia enterocolitica in slaughter pig tonsils: Enumeration and detection by enrichment versus direct plating culture. Food Microbiol. 27, 158-161, https://doi.org/10.1016/j.fm.2009.09.011

Vázlerová M., Steinhauserová I., 2006. The comparison of the methods for the identification of pathogenic serotypes and biotypes of Yersinia enterocolitica: Microbiological methods and PCR. Czech J. Food Sci. 24, 217-222

Wauters G., Kandolo K., Janssens M., 1987. Revised biogrouping scheme of Yersinia enterocolitica. Contrib. Microbiol. Immunol. 9, 14-21

Weynants V., Jadot V., Denoel P.A., Tibor A., Letesson J.J., 1996. Detection of Yersinia enterocolitica serogroup 0:3 by a PCR method. J. Clin. Microbiol. 34, 1224-1227 